\title{
Una committenza iberica nella Sicilia fra tardogotico e rinascimento
}

\author{
Marco Rosario Nobile
}

L'attività di mecenatismo e finanziamento artistico svolta dai Conti di Modica, Anna Cabrera e Fadrique Enriquez, nella cittadina di Caccamo, durante un breve soggiorno (1484), è registrata dallo storico siciliano Agostino Inveges ${ }^{1}$. La fonte segnala la volontà di ingrandire la chiesa madre dedicata a S. Giorgio e la realizzazione di una cappella funeraria nell'altare maggiore, un'opera che si prolunga per decenni, anche dopo la partenza definitiva dei Conti per la Spagna nel 1486. Dai pochi resti e frammenti della tribuna, conservati nella attuale chiesa (totalmente rifatta nella prima metà del XVII secolo) sembra evidente la scelta anticheggiante dei mecenati e l'utilizzazione di scultori in marmo attivi a Palermo, probabilmente la bottega di Domenico Gagini ${ }^{2}$.

Anche nel limitrofo castello di Caccamo si ricorda un'attività riedificatoria, attraverso la riparazione della torre del "Dammuso» e ulteriori interventi decorativi (affreschi datati al 1487). A questo periodo risale anche una lapide con scritta latina ( «Tempore felicil omnes gaudent amici〉 Dum fortuna perit $\backslash$ nemo amicus erit»), mentre cinquecenteschi, ma ancora riferibili alla volontà comitale, sono ulteriori interventi classicisti, come la cappella della SS. Concezione (1517) e una nuova porta del castello (1526) ${ }^{3}$.

Più decisamente orientata verso il gotico è la fondazione del convento di Francescani Osservanti intitolato a Santa Maria di Gesù a Modica ${ }^{4}$, da-

\footnotetext{
A. InVEges, La Cartagine siciliana, Palermo 1651, págs. 490-504.

H.W.: Krufr, Domenico Gagini und seine Werkstatt, München 1972, pág. 54, pág. 239, scheda n.12.

G. Lanza Tomasi, E: Sellerio, Castelli e monasteri siciliani, Palermo 1968, págs. 161-175.

4 Per la storia del complesso si rimanda in particolare a: F. RENDA, Prospetto corografico istorico di Modica di Placido Caraffa, Modica 1869 (ristampa, Bologna 1977), págs. 177-178, nota 41; E. MAURECl, La chiesa e il convento di Santa Maria di Gesù in Modica, in "L'Arte», XII (1909), fasc. VI, págs. 467-468; G. Bellafiore, Architettura in Sicilia (1415-1535), Palermo 1984, pág. 128.
} 
tata a partire dal 1478 , ma che risulta certamente correlata ai finanziamenti dei conti. II legato perpetuo concesso alla fabbrica sanciva il matrimonio svoltosi nel 1481 (forse a Modica), ed esprimeva la volontà del nuovo conte di risiedere nella Contea, come per obbligo dei capitoli matrimoniali ${ }^{5}$, probabilmente segnando anche una nuova direttrice di espansione della città verso l'altipiano. Una motivazione non secondaria nelle scelte dei finanziatori sembra poi affermare un preciso valore politico, dettato dall'atteggiamento apertamente antiebraico dei francescani, già determinante nella strage di Modica del 1474.

Basata su un rigido modulo «ad quadratum», la struttura venne realizzata nell'arco di circa mezzo secolo. Sicuramente le proporzioni dell'aula originaria dovevano essere di circa 1 a 3 , mentre l'altezza delle strutture murarie e le chiavi di volta (una di queste sembrerebbe portare la data del 1523), rintracciate durante i lavori di restauro, fanno intuire come la copertura delle campate fosse risolta con tre vaste crociere quadrate. L'inserimento di cappelle laterali a scopi prevalentemente funerari rientra in una generale nuova organizzazione delle chiese monastiche, a partire dalla fine del XIV secolo. Testimonianze documentarie della metà del XVI secolo attestano come la sepoltura in S.Maria del Gesù risultasse appetibile, nonostante la probabile saturazione degli spazi disponibili, per la creazione di nuove cappelle ${ }^{6}$.

Di particolare interesse è la cappella con ingresso esterno alla chiesa di Santa Maria di Gesù a Modica, basata su una volumetria cubica su cui doveva insistere una copertura particolare, forse una calotta su pennacchi come la cappella Cabrera in S. Maria di Betlem a Modica, mentre i contrafforti angolari potrebbero far pensare a una crociera "nuda» all'antica, come si realizzava nel 1538 a Ragusa nella cappella della Dormitio Virginis a S.Maria delle Scale ${ }^{7}$. Singolare è il portale, riferibile alla prima metà del XVI secolo: una atrofizzata cornice mistilinea tardogotica è incastrata da una edicola classicista. Fuori dagli schermi consueti è il timpano "a omega» (che potrebbe essere più recente), mentre i capitelli ionic:, con volute diagonali sembrano rivelare, nella fattura artigianale, una

\footnotetext{
5 Per i capitoli matrimoniali cfr.: R. SOLARINo, La Contea di Modica. Ricerche storiche, 2 voll., Ragusa 1885-1905 (ed. consultata, Ragusa 1981, págs. 164-171).

6 Archiv. di Stato Modica, notaio V. Cannella, atti del 30 novembre. 1557, 20 agosto 1558, 20 maggio 1559. L' Archivio di Stato di Modica non conserva documenti notarili della città anteriori al 1530 .

7 Per l'intervento in S. Maria delle Scale rimando al M.R. NOBILE, Architettura religiosa negli Iblei, Siracusa 1990, pág. 19. Non è da escludere che la cappella potrebbe essere messa in relazione alle esequie di Fadrique Enriquez, morto lo stesso anno a Medina de Rioseco.
} 
conoscenza di modelli classicisti, svolta eclusivamente attraverso disegni o incisioni.

La qualità artistica del prospetto chiesastico è stata generalmente riconosciuta. Basterà aggiungere come il corpo del portale richiami analoghe manifestazioni artistiche dell'area iblea. Si pensi alla Cappella della Candelora in S.Maria delle Scale a Ragusa e, in misura minore, al portale del S.Giorgio vecchio di Ragusa ${ }^{8}$. La larga strombatura e la fertilissima e brulicante decorazione con iconografie iperrealiste di fauna marina rientrano in una fase che crediamo compresa fra gli ultimi due decenni del xv secolo e i primi due del XVI. Messa sovente in relazione con la decorazione del levante iberico anche questa produzione rivela, a una più attenta analisi, caratteristiche diversificate e origini più complesse. II Mauceri, nella lunetta del portale registrava la firma di uno, molto più probabilmente, del principale artefice: "Man Ramunnu» ${ }^{9}$. Ramunnu doveva essere personaggio di grande prestigio e levatura per potersi permettere una firma nella lunetta del portale, non è poi sicuro se si tratti dell'ideatore dell'intero complesso o dello scultore della facciata.

Lo stesso tono di ricercatezza della facciata si riscontra nel chiostro quadrato, sorretto da colonnine decorate nel primo ordine e da pilastrini a base ottagonale nel secondo. La copertura del corridoio del primo ordine è risolta con eleganti crociere ancora in buono stato di conservazione. Al piano soprastante, il loggiato ha una copertura lignea che si appoggia alla muratura tramite mensole (sempre in legno), che riteniamo appartenenti ai primi anni del XVI secolo. Dei misteriosi quanto staticamente inutili pseudo-contrafforti poligonali segnano gli assi principali e gli angoli del chiostro.

L'uso di tozzi pilastri, collocati su un muretto e con archi a unico centro

8 Sul tardogotico nella Contea di Modica: E. MavCERI, La Contea di Modica ne l'arte, in “L'Arte», XVII (1914), fasc. II, págs. 120-134; G. AGNELLo, L'architettura aragonese-catalana in Italia, Palermo 1969, págs. 71-74; ID., Influencias y recuerdos españoles en Ragusa y su región, in «La huella de Espana en Sicilia», Revista geografica española, s.d., págs. 125-134. Si rinvia poi all'itinerario bibliografico contenuto in M. R. NOBILE, L'architettura nella Contea di Modica tra Quattro e Cinquecento, in «Bollettino della Biblioteca» (Università degli Studi di Palermo, Dipartimento di Storia e Progetto nell'Architettura), n. 1, págs. 49-52.

9 E. MAucERi, La chiesa e il convento..., cit.; A. VENTURI, Storia dell'arte italiana, Milano 1924, vol. VIII, parte II, pág. 89. Più che a mastro Ramunno da Burgos, il cognome Raimondi è da legare a ulteriori presenze siciliane come quella di Domenico Ramundo, probabilmente lombardo, che nel 1490 lavora nel palazzo Abatellis a Palermo (F. MELI, Matteo Carnilivari e l'architettura del Quattro e Cinquecento in Palermo, Roma 1958, doc. 14, pág. 225). Scultori o argentieri con lo stesso cognome si ritrovano ancora a Palermo nel 1532 (Abattista de Ramundo) (G. DI MARzO, op. cit., 2, doc. CCLVIII, pág. 329), e nel 1544 nella stessa Modica (Vincenzo de Ramundo) (ASM, notaio Pietro Di Giacomo, atto, 11 maggio 1544). 


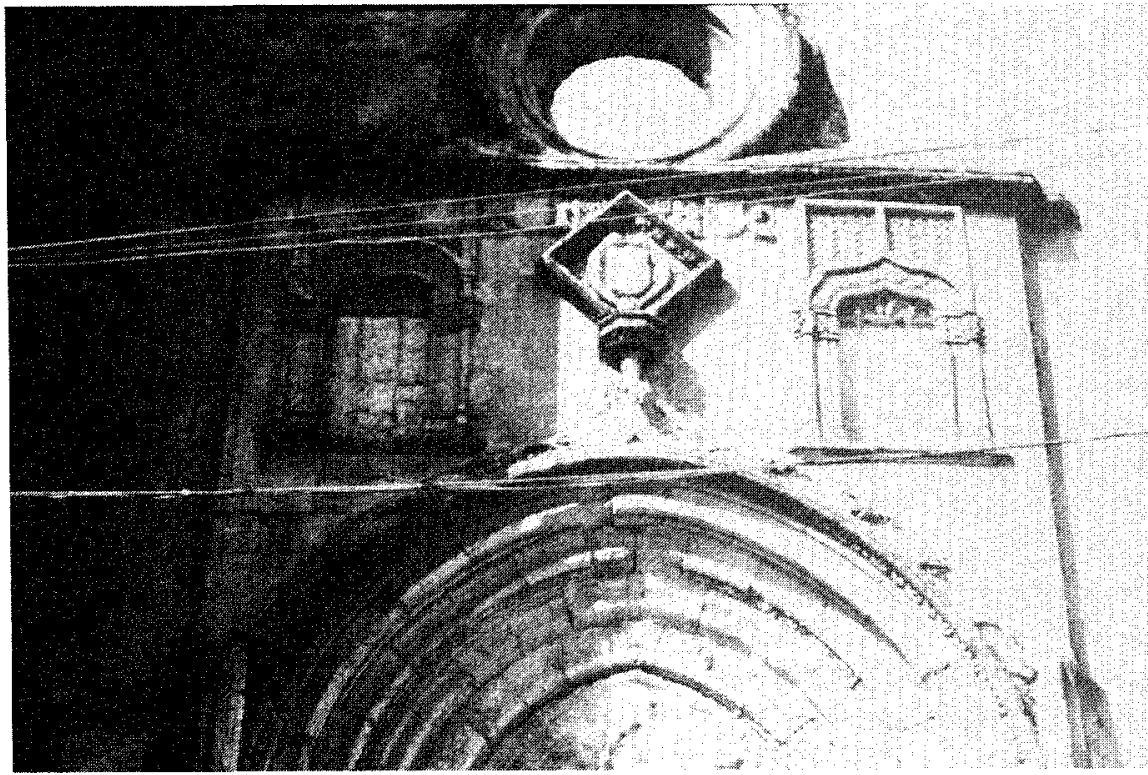

Modica. Chiesa di S. Maria del Gesū, particolare del prospetto.

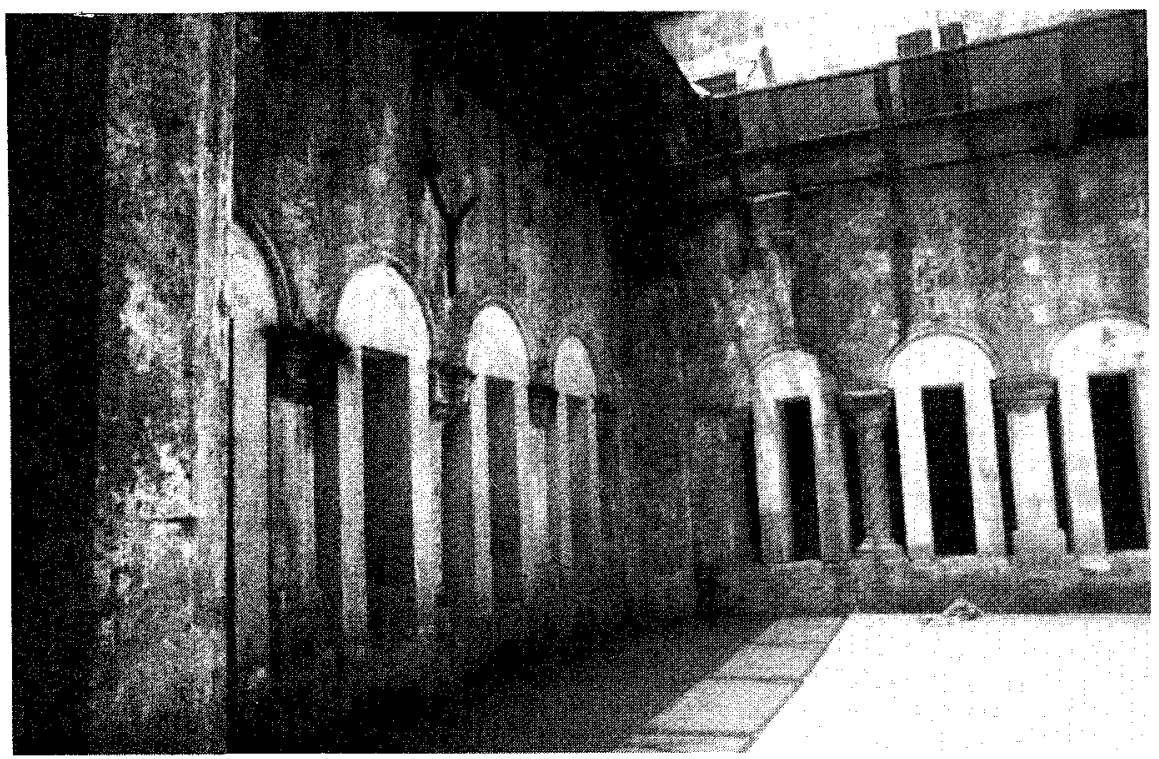

Modica. Convento di S. Maria del Gesū, chiostro. 
e sesto ribassato rientrano nel quadro di altre realizzazioni del secolo, come il chiostro di S. Maria del Gesù a Palermo ${ }^{10}$. Un modello analogo di pilastrino a base ottagonale si ritrova nei resti dei chiostri di S. Antonino e di S. Maria della Croce a Scicli (entrambe di Francescani, anche se non Osservanti) -opere entrambe del secondo-terzo decennio del Cinquecento e, nel caso di Scicli, ancora di diretto finanziamento comitale ${ }^{11}$.utili a datare la parte alta del loggiato. $E^{\prime}$ in realtà la ricerca distributiva del doppio loggiato (con le celle servite al piano superiore dal corridoio esterno) ad assumere una prima significativa differenziazione da altri esempi siciliani a noi noti. Doppi loggiati su «arcaici» pilastri ottagonali si ritrovano negli stessi anni (dall'ultimo ventennio del Quattrocento) in Lombardia, nel cremonese, per esempio nel complesso degli Osservanti di Santa Maria e Bernardino a Pianengo ${ }^{12}$. Anche qui come a Modica si tratta di una tipologia che non ha precedenti. Resterebbe da ipotizzare, ma è più di un'ipotesi, contatti e relazioni molto intensi con quanto si andava realizzando in altre aree, sopratutto dallo stesso Ordine, per la risoluzione di problemi distributivi e funzionali del convento ${ }^{13}$.

Per gli aspetti linguistici del complesso si è soliti fare riferimento a una corrente gotico aragonese-catalana, particolarmente viva nella Contea di Modica. Si tratta di una lettura utile a spiegare certi inserti scultorei. In realtà per la struttura architettonica del chiostro non è altrettanto facile instaurare relazioni con il mondo iberico: evidente, infatti, è la distanza con i chiostri catalani del Quattrocento dove le virtuosistiche forature delle pareti sono lontane dal tono cauto, seriale, arcaico che si riscontra a Modica. Sembrano invece palesi i richiami alla ripresa di una

10 Realizzato nella prima metà del Quattrocento: cfr. G. BRESC BAUTIER, Les ètapes de la construction de l'église de Santa Maria di Gesü hors de Palerme au xv siécle, in G. MotTA (a cura di), Studi dedicati a Carmelo Trasselli, Soveria Mannelli 1983, págs.145-156.

11 P. Nifosi', G. Drago, Aspetti storico artistici della Contea di Modica in S. Maria della Croce a Scicli, Scicli 1981

12 L. MAGGI, Le tipologie architettoniche dei conventi dell' "Osservanza" nel cremasco e cremonese, in II francescanesimo in Lombardia, Storia e arte, Milano 1983, cit., págs.403 -424.

13 Il protagonismo delle nuove fondazioni francescane nella Sicilia tra XV secolo e i primi decenni del successivo è indubbio. La rilevanza è stata riaffermata in un recente Convegno (Francescanesimo e civiltà siciliana nel Quattrocento, Palermo 25-31 ottobre 1992). Sull'architettura degli Osservanti in altre aree italiane rimandiamo a: M.SALMl, Bernardino, gli Osservanti e alcuni aspetti artistici del primo rinascimento in Toscana, in Bernardino predicatore nella società del suo tempo, Todi 1976, págs. 363-373; A. ScotT1, Architetture dei Francescani in Lombardia. Problemi e indicazioni di ricerca, in II francescanesimo in Lombardia,..., cit., págs.247-266. Si veda inoltre L. Di Fonzo, Francescani, in Dizionario degii Istituti di Perfezione, Iv, Roma 1977, ad indicem. L'autore cita (pág. 486) la Chronica fr. Minorum Observantiae (1480 c.) in cui si segnala la predilezione per fondazioni fuori dalle mura: "extra civitates et terras; nam nova loca sumendo nulli faciebant iniuriam,...". 
tradizione locale (le proporzioni contratte, la decorazione a spirale delle colonnine) di ascendenza normanna, tipica del primo Cinquecento isolano, con aperture alla tradizione di classicismo neo-romanico dell'Italia settentrionale (scelta dell'arco a tutto sesto, capitelli gotici sormontati da cornicioni seriali).

Legata ancora ai finanziamenti degli Enriquez-Cabrera è anche il complesso conventuale degli Osservanti ad Alcamo ${ }^{14}$, cittadina, situata tra Trapani e Palermo. Evidentemente, come già constatato per Caccamo, il ritorno in Spagna non interruppe le relazioni con gli ambiti siciliani. Sembra cioà di potere evidenziare ancora una committenza «a distanza», un ruolo che permette una gestione mediata delle scelte architettoniche.

Un tale aspetto risalta senz'altro per la chiesa (fondata nel 1477, ma probabilmente in costruzione solo dal $1484 \mathrm{ca}$.) dove si ravvisano soluzioni di cantiere non consuete in Sicilia. La provenienza da Genova delle colonne del portico (1512) rimanda a metodi poco frequenti per la prassi costruttiva dell'isola ${ }^{15}$, dove le affermate botteghe palermitane dei «marmorari» rispondevano bene alle esigenze di inserire nelle nuove fabbriche elementi architettonici classicisti (di solito in marmo bianco di Carrara). Ovviamente la scelta ricorda invece i criteri adottati da committenti spagnoli a partire dal famoso caso della Calahorra ${ }^{16}$. Non ancora risolto è un ulteriore precedente siciliano (ante 1487), al momento in cui gli scultori Gabriele di Battista e Antonio Mancino si impegnano a realizzare le colonne del cortile del nuovo palazzo Abatellis, con le stesse qualità e condizioni delle colonne del nuovo chiostro di S. Francesco a Palermo «construtti per Januenses" ${ }^{17}$. L'assenza di botteghe di marmorari genovesi a Palermo,

14 F.Rotolo, La chiesa di S.Francesco d'Assisi in Alcamo, Palermo 1977, pág. 21 nota 26; V. REGINA, Alcamo. Storia, arte e tradizione, I, Palermo 1980, págs. 81-82. Un portico su colonne che reggono una trabeazione rettilinea, integralmente rinascimentale, appare contemporaneamente anche nella poco nota chiesa francescana di S.Maria degli Angeli a Sant'Angelo di Brolo (post 1506), in un ambito geografico dipendente da Messina e sicuramente più aperto alle influenze continentali. Se ne veda la scheda in: C. Sinoti, Architettura, luogo e memoria, S. Angelo DI BROLO 1990.

i5 Le notizie sul cantiere di $\mathrm{S}$. Maria del Gesù ad Alcamo sono tratte dalle preziose annotazioni documentarie di padre Rotolo (vedi nota precedente).

16 Rimandiamo in particolare a: H. W. KRUFT, Un cortile rinascimentale italiano nella Sierra Nevada: La Calahorra, in "Antichità viva», XI, 1, 1972, págs. 35-45; V. NiETO, Renovación e indefinición estilistica, 1488-1526, in V. Nieto, A. J. MORALES, F. CHECA, Arquitectura del Renacimiento en España, 1488-1599, Madrid 1989, págs. 13-96, alle págs. 44-51; F. MARIAs, El largo siglo XVI, Madrid 1989, págs. 260-261, pág.380; ID., Sobre el castillo de La Calahorra y el Codex Escurialenses, in C. Bozzon, G. Carbonara, G. VILLetI, Saggi in onore di Renato Bonelli, I, Roma 1992, págs. 539-553, con ulteriore bibliografia. 
farebbe pensare ancora a una spedizione di materiali dal capoluogo ligure, probabilmente concorrenziale alle attivissime botteghe palermitane (si pensi che già nel 1484 Domenico Gagini aveva fornito le colonne e i fastosi capitelli della chiesa dell'Annunziata a Palermo e che nel 1487 spediva quattordici colonne e capitelli a Messina per il chiostro di S. Francesco ${ }^{18}$ ). II metodo degli elementi pre-scolpiti, a causa dei delicati equilibri di cantiere, risulta, comunque, essere corrente nelle fabbriche siciliane.

Il cantiere della chiesa di S.Maria del Gesù ad Alcamo si concludeva entro un decennio se nel 1519 veniva impegnato lo scultore Jacobus La Porta da Bologna, per la realizzazione del coro ligneo ${ }^{19}$.

A date più tarde (1533), la presenza di un maestro Gerbasio de Lugo a cui si erano obbligati come fornitori di materiali lapidei i maestri Filippo lo ludichi e Antonio de Modica (è presumibile che si tratti di maestri provenienti dalla Contea) farebbe intuire l'affidamento della costruzione del convento e chiostro e la messa in opera a un capomastro non siciliano, anche se, come di regola, "cives Panormi», cioè abitante a Palermo, in ossequio alle rigide regole corporative dei «fabricatores» ${ }^{20}$. La frequenza di operatori esterni, e probabilmente di prestigio, deve far riflettere, sebbene nell'intreccio tra committenza e finanziatori risulti sfuggente il ruolo svolto dai Conti. Se la lontananza farebbe optare per una maggiore autonomia decisionale dei francescani, le scelte anticheggianti sembrano tuttavia confermare un'attenzione diretta. E' il quadro detto comunemente della "Madonna Greca», dove compaiono i Conti di Modica, a svelare così un intento celebrativo moderno, teso all'esaltazione dei finanziatori.

Si ricorderà qui come dalle ricerche di Miguel Falomir Faus risulti che l'Almirante di Castiglia e la contessa di Modica abbiano intessuto relazioni con Alonso de Berruguete e con altri artisti ${ }^{21}$. Diventerebbe così da rive-

17 G. DI MARzo, I Gagini e la scultura in Sicilia nei secoli xv e xvI, 3 voll. Palermo 1880/83, 2, doc. n. VIII, págs. 10-11. Padre Rotolo, tuttavia, segnala un Filippo lanuensis che intorno al 1480 lavora nel convento di S. Francesco (F. Rotolo, La Basilica di S. Francesco di Assisi in Palermo, Palermo 1952, pág. 114, nota 49). E' possibile, infine, che lo stesso Domenico Gagini possa essere stato considerato, per i suoi trascorsi di lavoro, come genovese.

18 H. W. KRUFT, Domenico Gagini..., cit., pág. 40 e pág. 271 docc. nn. xxXVIII-XXXIX.

19 G. DI MARzO, op. cit., 2, doc. n. cccx, págs. 397-398.

20 Sulle problematiche relative alla corporazione dei "fabricatores" nota attraverso un documento pubblicato dal Di Marzo ( G. DI MARzo, op. cit., 2, doc. IV, págs. 4-7). Si veda poi B. PATERA, "Marmorari e muratori" nel Privilegium del 1487, in IMestieri. Organizzazione, tecniche, linguaggi, Palermo 1984, págs. 199-222. In corso di stampa è il mio contributo: M.R. NobILE, /I Tardo Gotico. Note sul cantiere siciliano tra XV e XVI secolo, presentato in occasione del Convegno di Studi in «L'architettura del Tardogotico in Europa», Milano, 21-22-23 febbraio 1994. 


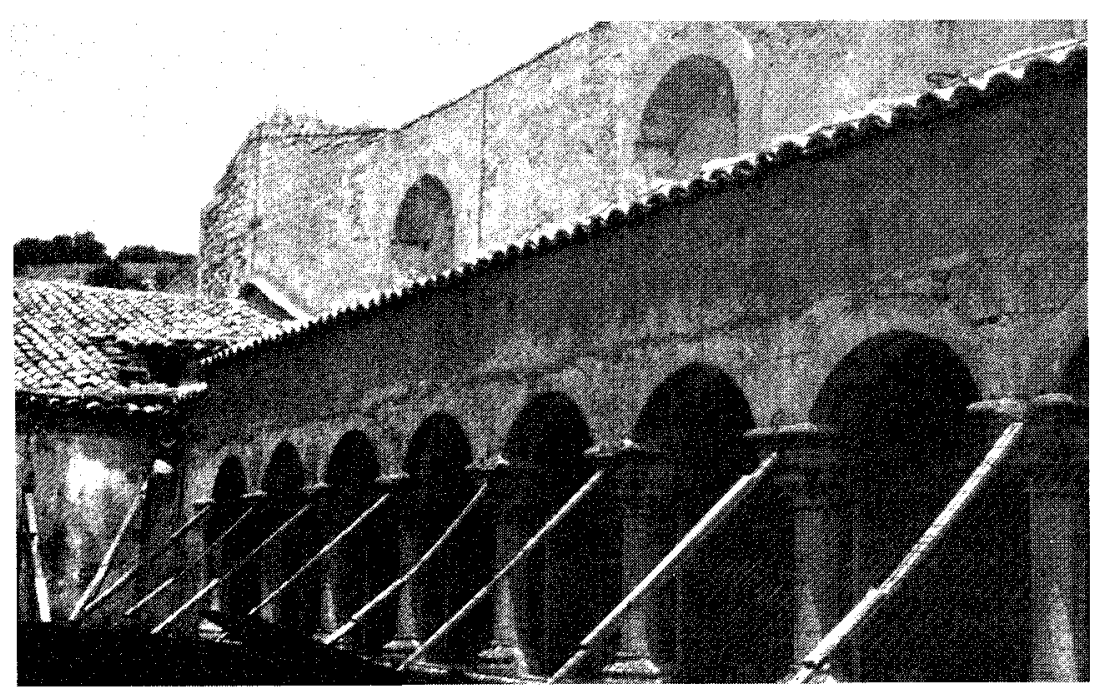

Modica. Convento di S. Maria del Gesū, chiostro, loggiato superiore.

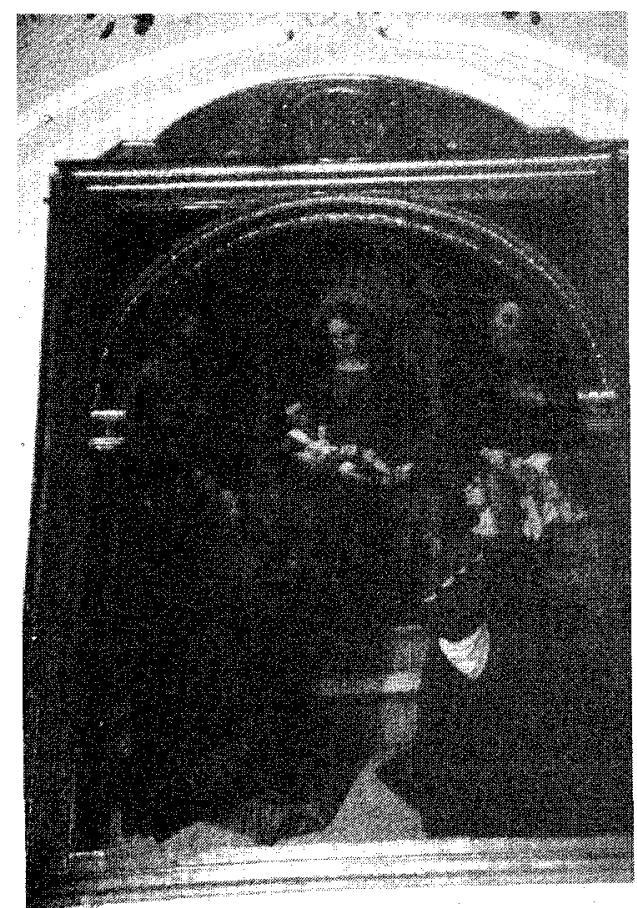

Alcamo. Chiesa di S. Maria del Gesü, «Madonna Greca», in basso i Conti di Modica. 
dere l'attribuzione a Pier Francesco Sacchi del dipinto di Alcamo ${ }^{22}$ in funzione di un più attento controllo degli artisti gravitanti nel primo Cinquecento negli ambiti di Valladolid o di Medina de Rioseco e sottoponendo agli specialisti l'ipotesi di una personalità di alto livello.

La storiografia artistica ha sovente sottolineato la stretta dipendenza della produzione architettonica siciliana tra tardogotico e rinascimento con la coeva civiltà spagnola. Dalla stessa Spagna sono state, sino a tempi recenti, traslate le categorie storiche atte a spiegare le presunte successive tappe del fenomeno, le sue mutuazioni ibride nella direzione del classicismo, predisponendo cioè la sequenza: gotico aragonese-catalano, plateresco, rinascimento ${ }^{23}$.

Una più attenta verifica dei fenomeni di sincretismo, la sempre meno decisa definizione dei confini del mondo rinascimentale, lo stesso dibattito sul plateresco hanno probabilmente reso obsolete queste letture ${ }^{24}$. Ad atteggiamenti astrattamente pregiudizievoli vanno del resto ricondotte le linee interpretative esclusivamente filo iberiche e, sul fronte opposto, quelle portate a una sopravvalutazione degli aspetti «italianeggianti» della produzione isolana ${ }^{25}$.

II ruolo della Spagna in questi anni cruciali investe vari livelli, si pensi al nucleo di operatori attivi a Palermo alla fine del xv secolo, alle committenze come quella dei Bonnett o dei Talamanca nella Palermo degli anni settantaottanta del XV secolo ${ }^{26}$, alla molteplice e capillare presenza di aristocratici, notabili e religiosi, che, come invece superficialmente si è portati a pensa-

21 M. Falomir Faus, Alonso Berruguete, Leonardo da Vinci y un episodio temprano de la "querelle» en España, in "Archivo Español de Arte», 262 (1993), págs. 181-184. Sull'attività di mecenatismo svolta in Spagna da FADRIQUE ENRIQUEZ si veda anche F. MARIAS, El largo..., cit., págs. $118,126,159,318$.

22 R. LoNGHI, Frammento siciliano, in «Faragone» IV, 47, 1953, págs. 3-44, ripubblicato in Edizione delle opere complete di Roberto Longhi, I, Firenze 1975, págs. 143- 175, alla pág. $172 ;$ V. Aвbate, Revisione di Antonello il Panormita, in "BCA Sicilia», III, 1-2-3-4 (1982), págs. 39-68, alla pág. 66 nota 35.

${ }_{23}$ Una lettura di questo genere, benché scevra da tentazioni evoluzionistiche, compare ancora in uno dei più recenti contributi monografici sull'architettura siciliana tra Quattro e Cinquecento: $G$. BELLAFIORE, op. cit, in particolare págs. 97-104. Si veda tuttavia come in un suo fondamentale saggio Maria Giuffrè abbia contestato l'uso della categoria «plateresco siciliano": cfr. M. GiUfFRE', Architettura e decorazione in Sicilia tra Rinascimento, Manierismo e Barocco 1463-1650, in "Storia Architettura», 1-2, 1986, págs. 11-40, in particolare pág. 20.

24 J. B. BurY, The stylistic terme "Plateresque", in "Journal of the Warburg and Courtauld Institutes», 39 (1976), págs. 199-230; F. MARIAS, La arquitectura del Renacimiento en Toledo (1541-1631), I, Toledo 1983, págs. 22-28; V. NIETO, op. cit., págs. 58-65.

25 Una utile guida bibliografica su questi temi si può trovare in M.S. DI FEDE, Gli studi sull'architettura del primo rinascimento in Sicilia, in "Bollettino della Biblioteca", (Dipartimento di Storia e Progetto nell'architettura, Palermo), 1, 1992, págs. 39-48.

26 Sul cantiere palermitano del Quattrocento e sul ruolo delle maestranze iberiche: F. MELI, op. cit., in particolare págs. 65-79; G. BRESC BAUTIER, La «maramma» de la cathédrale de Palerme aux 
re, non sono assolutamente chiusi o ostili al linguaggio classico in nome di un estremismo nazionalistico. In realtà risulta problematico, a partire dalla committenza nobiliare attiva in Sicilia, ritagliare una specifica identità spagnola da contrapporre a un partito italiano. Si assiste piuttosto a un atteggiamento eclettico, che, in generale, non disdegna di realizzare edifici gotici, ma apprezza e predilige la scultura rinascimentale e indirizza il rigido mondo del cantiere siciliano all'assorbimento del classicismo, alla pratica corrente dell'inserto anticheggiante.

La registrazione unanime di una enclave aragonese-catalana nell'estremo sud dell'isola sembra fondarsi tuttavia proprio su una committenza politicamente ed etnicamente orientata. Le città della Camera Reginale, sopratutto Siracusa, e i centri della Contea di Modica, retta dai Cabrera, spiegherebbero la persistenza e l'uso continuato di tipologie e linguaggi del levante iberico. Da Noto sarebbe pervenuto a Palermo il maggior architetto siciliano del tempo: Matteo Carnilivari ${ }^{27}$, e sono numerosi gli operatori di quest'area attivi nel cantiere palermitano del Quattro e Cinquecento. Accanto all'esportazione di tipologie (in realtà non totalmente verificabile) è la radicata tradizione dell'intaglio lapideo a fornire una motivazione alla mobilità delle maestranze.

La breve presenza siciliana di Fadrique Enriquez e della consorte si colloca dopo la prima ondata di classicismo rinascimentale che aveva investito l'isola. II grande sisma del 1693 ha potenzialmente distrutto numerose testimonianze, ma certamente in ambiti prossimi a Modica, nella colta città di Noto la famiglia degli Speciale aveva realizzato alla metà del XV secolo, -probabilmente in anticipo all'arrivo in Sicilia di Domenico Gagini e Francesco Laurana e quindi attraverso l'importazione di marmi pre-scolpiti (dalla Toscana?)- una tomba in S. Francesco ${ }^{28}$. La magnifica committenza di Pietro Speciale avrebbe commissionato a Domenico Gagini la costruzione di una cappella gentilizia nella cattedrale (cappella di $S$. Cristina) e nella chiesa di S. Francesco a Palermo ${ }^{29}$, dove fra 1460 e 1480 si erano succedute le più innovative realizzazioni di cappelle funerarie, commissionate da potenti famiglie (De Chirco, Alliata, Mastrantonio,

XIV e XV/ siècles, in "Commentari», ns., 27 (1976), fasc. I-II, págs. 109-120 (per l'artista catalano Nicolaus Comes).

${ }_{27}$ Su Matteo Carnilivari, oltre al già citato testo del Meli, si segnala la monografia di: $F$. Rotolo, Matteo Carnilivari. Revisione e documenti, Palermo 1985.

28 H. W. Kruft, Domenico ..., cit., pág. 28, pág. 259, scheda A7; F. Rotolo, La chiesa di S. Francesco d'Assisi a Noto, Palermo 1978, págs. 39-46.

29 F. Rotolo, La Basilica ..., cit., págs. 97-114. 
Galletti). Problematica e da decifrare è anche la committenza filo-classicista dei Cabrera su cui esistono validi indizi come il fonte battesimale, opera secondo il Kruft di Domenico Gagini, datato 1466 (con insegne dei Cabrera e dei Prades) conservato a S.Giorgio a Caccamo e donato alla chiesa da Bernardo II Cabrera ${ }^{30}$. Della stessa tomba di Bernardo I e Bernardo II nell'altare maggiore di S. Giorgio a Ragusa (1466) si conserva (attualmente nel S. Giorgio nuovo) un frammento anticheggiante con scritta in lettere latine e nella chiesa si trova anche una ignorata statua di Madonna, che probabilmente doveva far parte del cappellone quattrocentesco. Scomparsa è la tomba di Giannotto Cabrera nell'altare maggiore del S.Pietro di Modica ${ }^{31}$, in una fase (anni settanta del xv secolo) in cui la sepoltura classicheggiante era oramai considerata dalle élites nobiliari siciliane segno di bon-ton.

C'è da chiedersi quanto abbiano pesato queste esperienze siciliane nel futuro Almirante di Castiglia e che ruolo può avere avuto l'ambiente culturale tutt'altro che periferico della scuola umanistica di Noto, nello sviluppare i germi di un gusto all'antica, che sarebbe emerso anche in anni successivi. La notizia, segnalata dal Trasselli ${ }^{32}$, che con Fadrique Enriquez e Anna Cabrera si sposterà in Spagna l'intellettuale Lucio Marineo Siculo, più tardi storiografo di Ferdinando il Cattolico e professore a Salamanca, è la spia di un mecenatismo a vasto raggio, aperto alle più nuove correnti culturali. Accanto alla legittima domanda sul ruolo dei conti di Modica in Sicilia, bisognerebbe quindi interrogarsi su quanto la situazione siciliana è riuscita a cambiare i committenti, mettendoli a confronto, all'alternativa italiana al gotico internazionale, e a un circuito di raffinati intenditori e intellettuali che avevano aperto le porte agli scultori classicisti, reduci dal cantiere dell'Arco di Castelnuovo. II fenomeno non è isolato, né circoscrivibile solo a sparuti esempi. In realtà sfugge ancora sggi la dimensione cortese del primo rinascimento siciliano, il fenomeno che indusse a una rinnovato interesse urbano, alla creazione di una committenza raffinata e colta che avrebbe decretato il successo (tuttora largamente inspiegabile) di un Domenico o di un Antonello Gagini e la creazione e proliferazione di effimeri centri di cultura. Assoro dei Valguarnera, Comiso dei Naselli, Pietraperzia dei Barresi sono solo alcuni casi che meritano studi non

30 H. W. Kruft, Domenico ..., cit., pág. 54 e pág. 238, scheda n. 11.

31 F. RENDA, op. cit., pág. 73.

32 C. Trasselli, Da Ferdinando il Cattolico a Carlo V. L'esperienza siciliana 1475-1525, Soveria Mannelli 1982, pág. 7. 


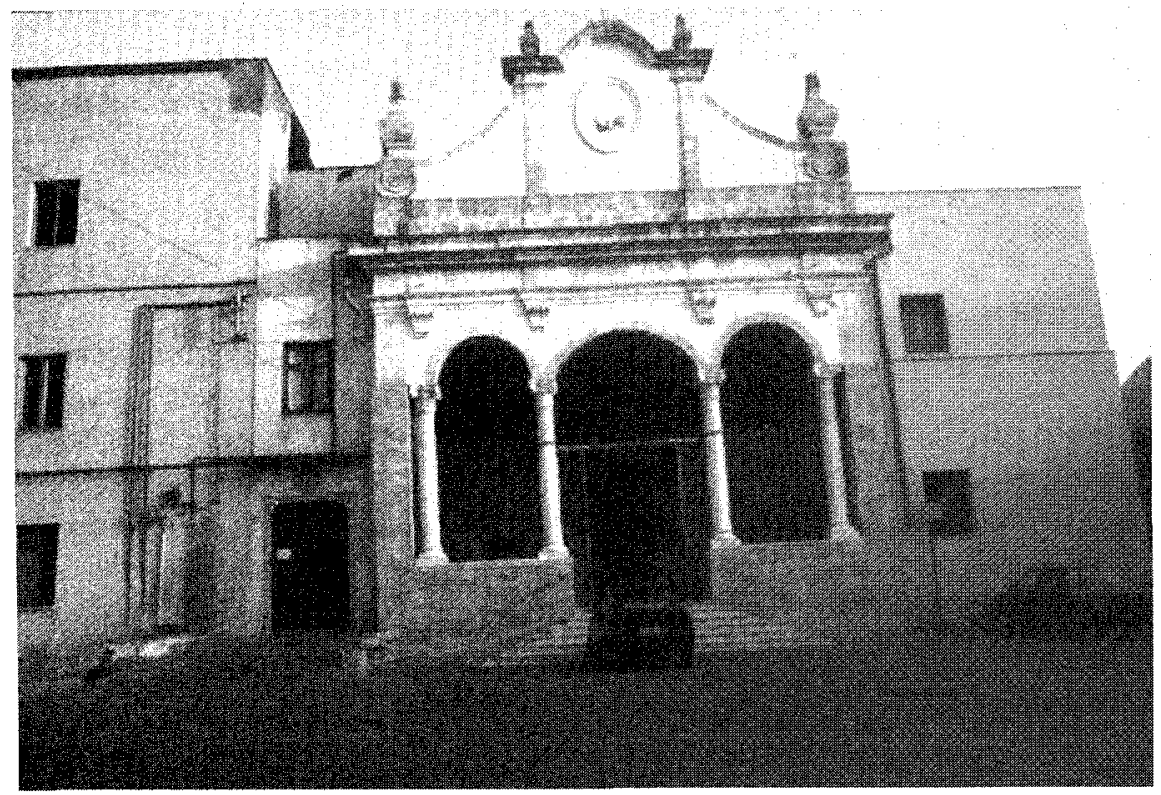

Alcamo. Chiesa di S. Maria del Gesū, prospetto.

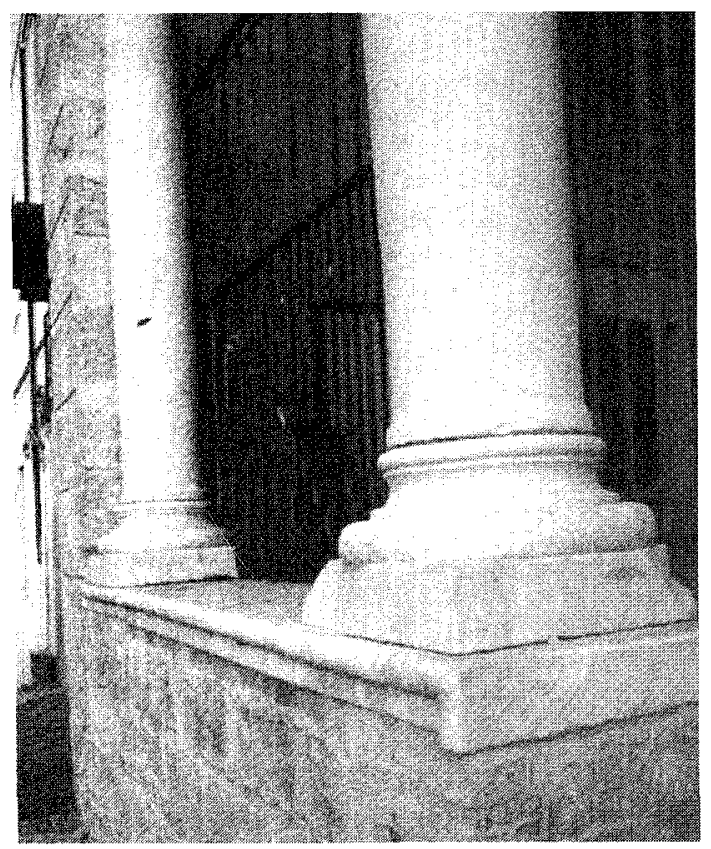

Alcamo. Chiesa di S. Maria del Gesū, particolare delle colonre del portico. 
ancora affrontati; mentre da valutare storicamente è il peso che la crisi economica degli inizi del XVI secolo e soprattutto la repressione dopo i tentativi di sommossa del 1516-1523 hanno avuto nel soffocamento o nel rallentamento di queste esperienze.

Per la grande Contea di Modica gli aspetti irrisolti sono ancora preponderanti, e tali da coinvolgere altri grandi complessi. Quale funzione possono avere svolto i Conti nella costruzione della chiesa madre di Scicli, andata distrutta, ma che una lastra datava al 1489, citando il fabricatore Guilelmus Belguardo? ${ }^{33}$ Quale funzione nelle prime esperienze classiciste?

Nell'ottica dell'ibrido ma con una apertura straordinaria verso il mondo rinascimentale è il convento di Francescani Conventuali di S. Antonino a Scicli ${ }^{34}$, la cui fondazione oscillerebbe tra 1514 e 1522. La costruzione di una cappella funeraria che funge da tribuna, coperta a cupola costolonata, ma con inserti classicisti deve necessariamente essere accostata a una committenza alta, che attualmente non è nota, ma che potrebbe essere stata determinante per costruzioni di cappelle di corte come quelle di Comiso (voluta dai Conti Naselli nel 1517) o di Militello ${ }^{35}$ : cappelle cupolate aggregate come tribuna a chiese francescane, secondo la consuetudine inaugurata dal progetto dell'Alberti per il Tempio Malatestiano. Indubbiamente la cappella (attualmente in pessimo stato di conservazione) assume un valore competitivo tanto da potere essere messa in relazione solo con iniziative comitali. La cappella "Cabrera» in Santa Maria di Betlem a Modica assume un significato analogo, ancora più ricco e celebrativo; fermo restando che la sua costruzione deve riferirsi ai primi decenni del XVI secolo, si deve ancora pensare a una committenza alta, forse un ramo della famiglia dei Cabrera. Si tratta di opere che non è possibile leggere con gli schematismi

33 P. Nifosi", La chiesa di S. Matteo a Scicli, in "La Provincia di Ragusa», 5, 1992, inserto.

34 ID., In rovina il convento e la chiesa di Sant'Antonino, in "Il Giornale di Scicli», 22 maggio 1983. Sul fenomeno delle cappelle a pianta quadrata, cupolate si fa riferimento a M. GIUFFRE', $L e$ "cappelle a cupola su nicchie" fra tradizione e innovazione, comunicazione presentata al Seminario su "Matteo Carnilivari e l'architettura siciliana fra Quattro e Cinquecento", Palermo, dicembre 1985, di prossima pubblicazione in "Storia architettura", e il cul manoscritto mi è stato gentilmente fornito dall'autrice.

35 F. Rotolo, Comiso, la chiesa di S. Francesco d'Assisi, Palermo 1981, págs. 23-34; M.R. NobILE, Architettura religiosa negli Iblei. Dal Rinascimento al Barocco, Siracusa 1990, págs. 19-22; G. Pagnano, Un organismo centrico della Maniera. Rilievo della cappella di S. Antonio di Padova in Militello Val di Catania, in "Quaderno dell'ist. Dipart. di Architettura ed Urbanistica Univ. di Catania", 13 (supplemento) 1983.

35 M. R. NoblLe, La cappella Cabrera di Modica, in "Kalos», 5, 1993, págs. 26-29. 
di un mitizzato e rigido universo classicista poiché esplorano una via siciliana, un «antico» autoctono e pervengono a un rinascimento esotico che affonda le radici in tecniche costruttive locali.

I grandi passaggi nodali che gettano ponti tra l'ultimo gotico e il rinascimento siciliano seguono probabilmente vie e vicende differenti dal contemporaneo travaglio iberico, ma altrettanto complesse e non sottovalutabili sono le strade di un interscambio culturale stretto. Alla constatazione di comunanze linguistiche, di semplici forme, va anche affiancata una ricerca senza pregiudizi che tenga in giusta considerazione la mobilità della committenza, i suoi programmi e le sue idee. 